\title{
ErasmusMC COMBINED EFFECTS OF SIROLIMUS AND MITOTANE IN THE INHIBITION OF GROWTH OF HUMAN ADRENOCORTICAL CARCINOMA CELLS
}

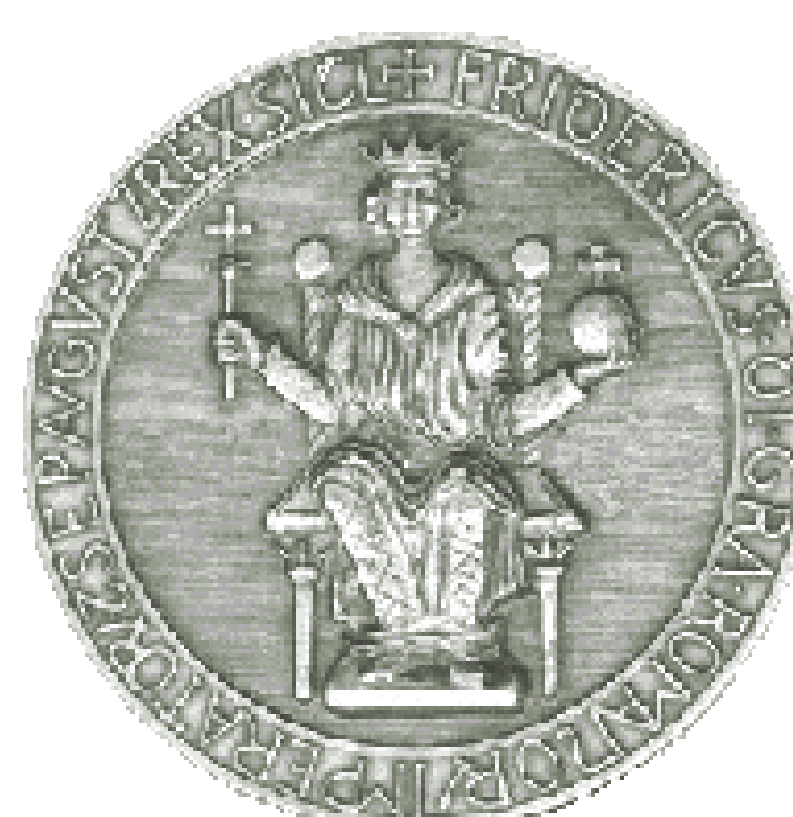

De Martino M.C. ${ }^{1}$, van Koetsveld P.M. ${ }^{1}$, Feelders R.A. ${ }^{1}$, Lamberts S.W.J. ${ }^{1}$, de Herder W.W. ${ }^{1}$, Colao A. ${ }^{2}$, Pivonello R. ${ }^{2}$, Hofland L.J. ${ }^{1}$

${ }^{1}$ Department of Internal Medicine, Division of Endocrinology, Erasmus MC, Rotterdam, Netherlands.

${ }^{2}$ Dipartimento di Medicina Clinica e Chirurgia Endocrinologia e Metabolismo Università degli Studi di Napoli Federico II, Naples, Italy

\section{BACKGROUND}

Adrenocortical cancer (ACC) is a rare cancer with poor prognosis and scant treatment options. Mitotane alone, or in combination with cytotoxic chemotherapy, represents the referral current treatment for patients with unresectable ACC. Recent studies have shown that mTOR inhibitors suppress growth of ACC cells.

AIM

This study aimed at evaluating the effects of mitotane in combination with sirolimus, an mTOR inhibitor, in ACC cell lines.

\section{METHODS}

In H295 and SW13 cells we tested the effects of a 6 day treatment with increasing doses of mitotane in the presence or absence of selected doses of sirolimus on cell proliferation as measured by the total DNA content. The tested doses of mitotane ranged between $10^{-7} \sim 10^{-5} \mathrm{M}$ in both $\mathrm{H} 295$ and SW13 cells, sirolimus was tested at concentrations of $5^{*} 10^{-9}$ and $10^{-}$ ${ }^{6} \mathrm{M}$ in $\mathrm{H} 295$ and $5^{*} 10^{-11} \mathrm{M}$ and $10^{-10} \mathrm{M}$ in SW13.

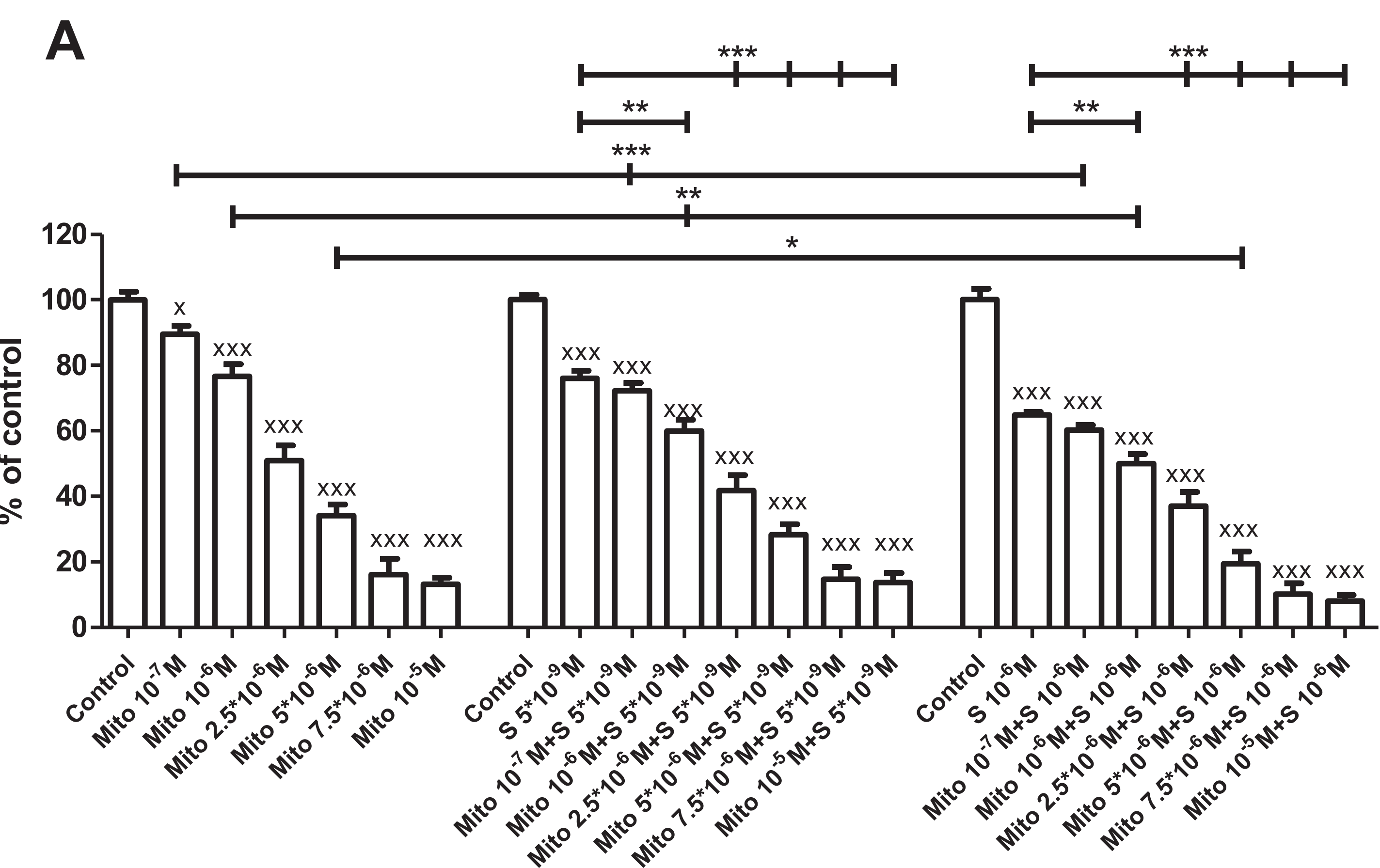

B

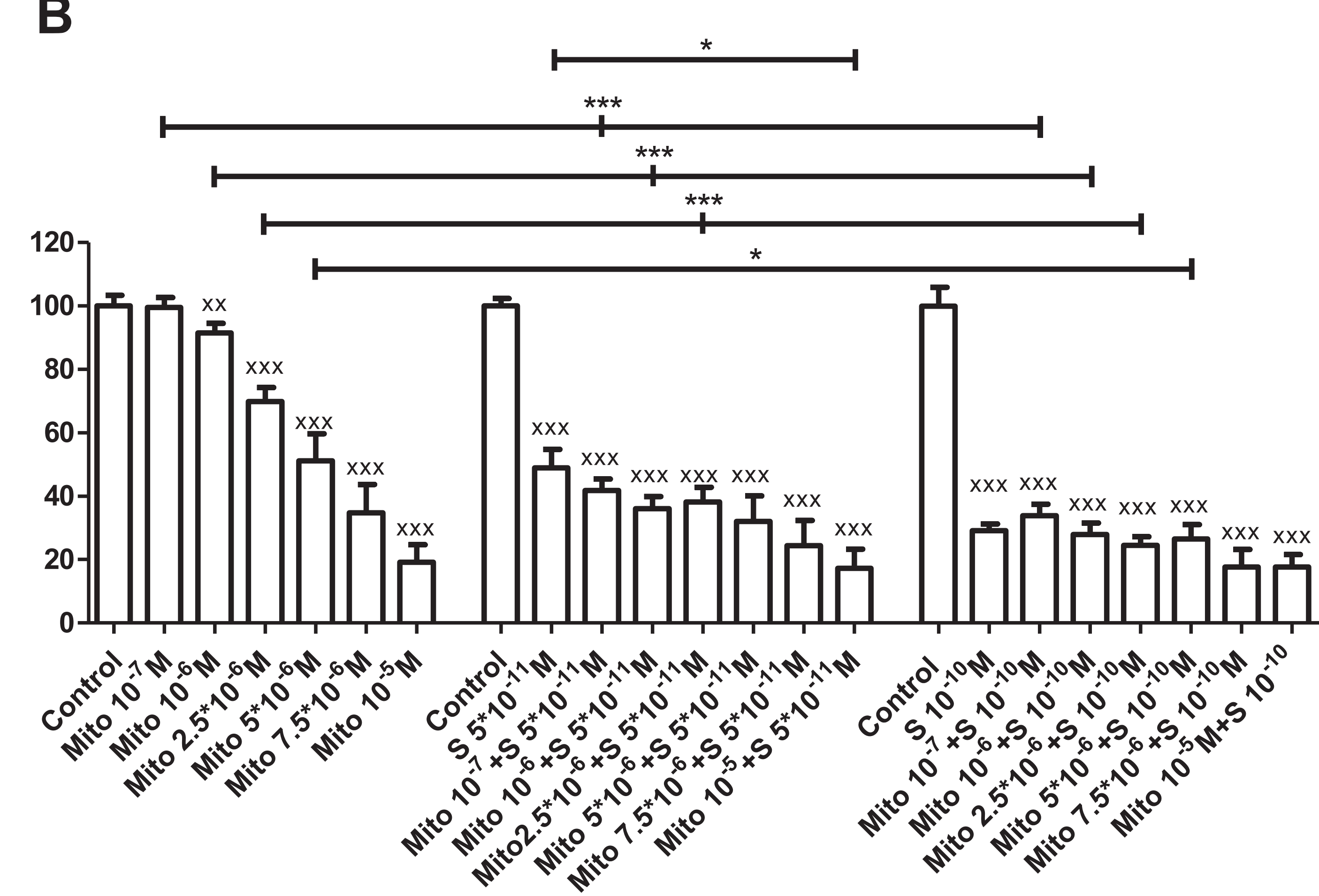

Figure 1. Combined effects of a 6 day treatment with increasing concentrations of mitotane (Mito) and selected concentrations of sirolimus (S) in two human ACC cell lines: H295 (panel A) and SW13 (panel B). At some of the combinations tested significant additive inhibitory effects on cell growth are observed in both cell lines. ${ }^{*} p<0.05,{ }^{* *} p<0.01$ and ${ }^{* * *} p<0.001 ; x p<0.05$, $\operatorname{xxp}<0.01$ and $\operatorname{xxxp}<0.001$ vs control.

\section{RESULTS}

$>$ In H295, mitotane significantly inhibited cell proliferation at all concentrations tested, with an IC50 of $4.5^{*} 10^{-6} \mathrm{M}$ and a maximal inhibition of $87 \%$ as compared with vehicle-treated controls $(p<0.001)$

$>$ In SW13, mitotane significantly inhibited cell proliferation at concentrations higher than $2.5 \times 10^{-6} \mathrm{M}$, with an IC50 of $1.6 \times 10^{-5} \mathrm{M}$ and a maximal inhibition of $81 \%$ as compared with vehicle -treated controls ( $p<0.001)$ (figure 1).

$>$ In both H295 and SW13 sirolimus significantly inhibited cell proliferation at both concentrations tested and when combined with mitotane, it showed statistically significant additive effects. This additivity was observed only with low mitotane doses (between $10^{-7} \sim 5^{*} 10^{-6} \mathrm{M}$ ) (figure 1 ).

$>$ Using mitotane doses higher than $5^{*} 10^{-6} \mathrm{M}$ the cell proliferation inhibition was already nearly maximal and no significant additive effects could be observed (figure 1).

$>$ The addition of sirolimus to mitotane did not significantly change the dose response curve of mitotane (figure 2).

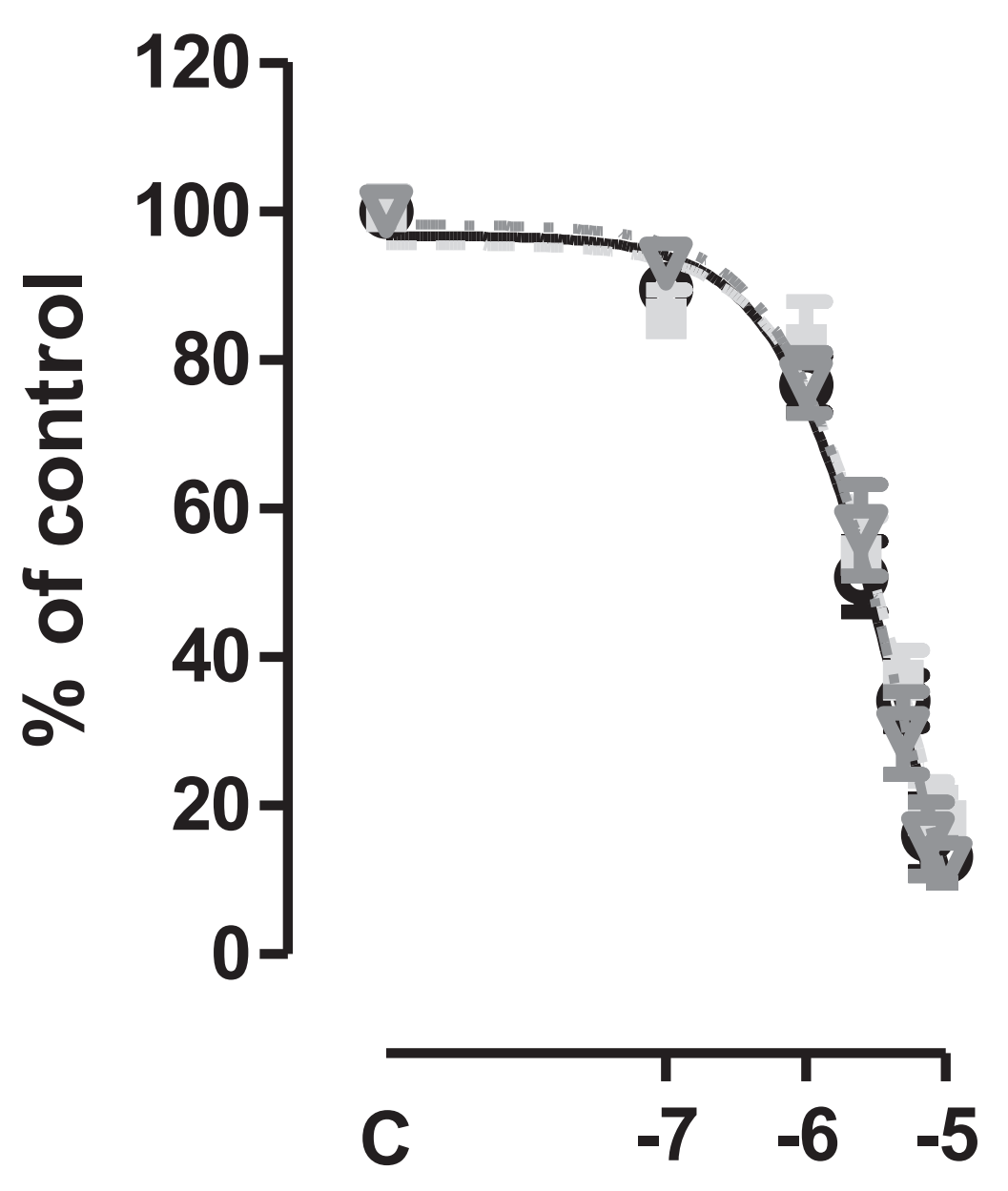

$\log$ [mitotane] (M)

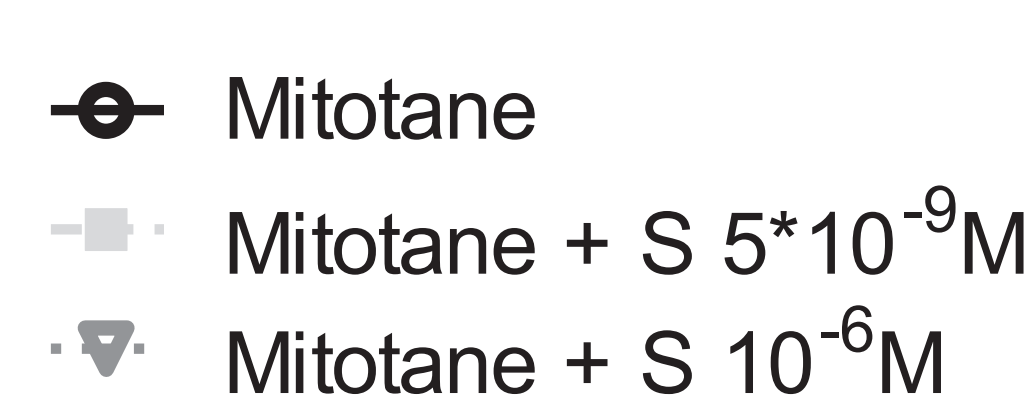

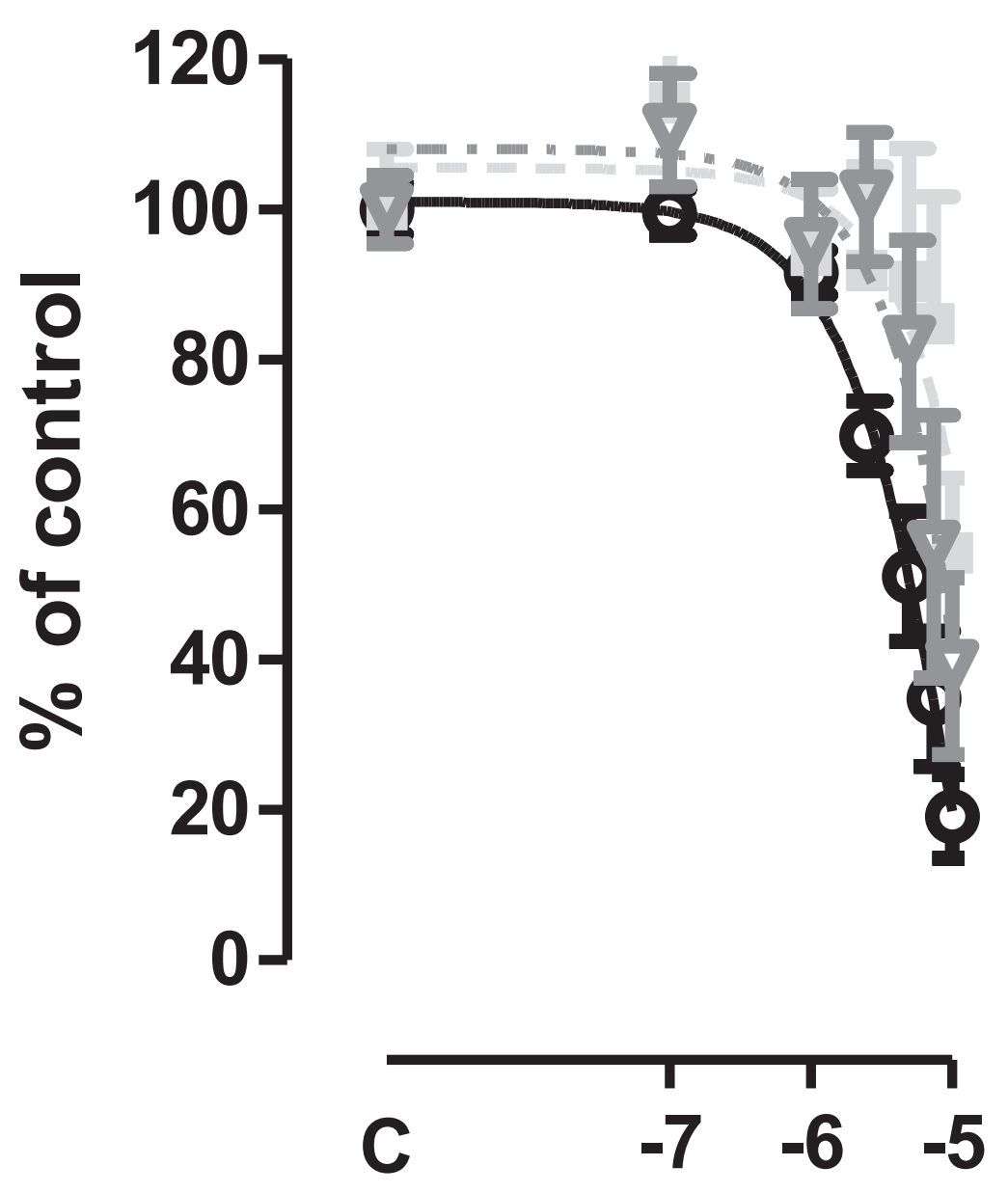

$\log$ [mitotane] (M)

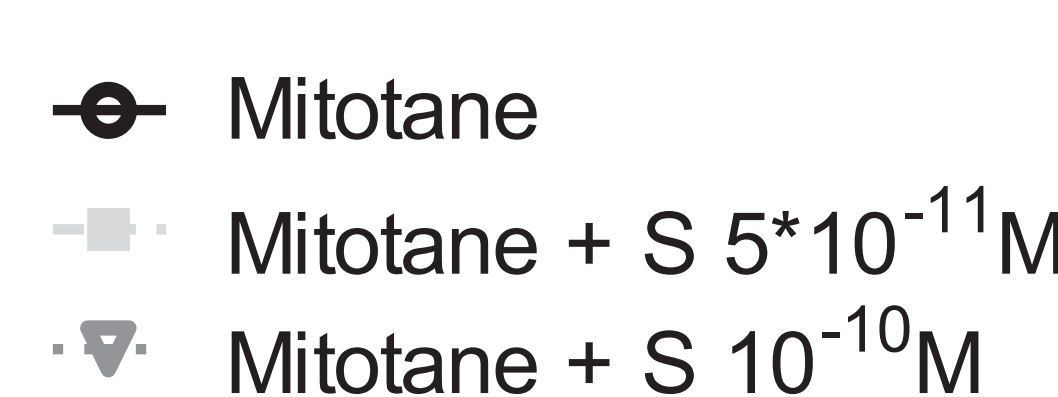

$\cdot \nabla$. Mitotane $+S 10^{-10} \mathrm{M}$
Figure 2. Dose-response curve of 6 days treatment with increasing mitotane M) concentrations, alone or in combination with two selected concentrations of sirolimus (S), in two ACC cell lines: H295 (panel A) and SW13. The addition of sirolimus to mitotane did not significantly change the dose response curve of mitotane. Data are expressed as the percentage of control and represent the mean \pm SD. Control is set as $100 \%$ and in the curve of mitotane alone the control is represented by vehicle-treated cells, whereas in the curves of mitotane combined with sirolimus the control is represented by treated cells with sirolimus alone.

\section{CONCLUSIONS}

The current study demonstrates that sirolimus has additive antiproliferative effects when combined with low mitotane doses. These doses correspond to concentrations lower than the therapeutic range of mitotane. If this effect can also be achieved in vivo, our data suggest that the addition of sirolimus to mitotane might be useful in ACC patients when the therapeutic range of mitotane range is not reached. 